\title{
SUR CERCARIA OCELLATA LA VALETTE
}

\author{
Par Paul MATHIAS
}

Les trématodes parasites dans les vaisseaux sanguins des vertébrés supérieurs (mammifères et oiseaux) ont été rencontrés peu souvent en Europe. Il en a été signalé :

En Europe, par Kowalewski (1895-96) (Bilharziella polonica Kow.) ;

En Russie, par Skrjabin (1920-1924) (Trichobilharzia kossarewi Skrjabin et Zakharow, Dendritobilharzia pulverulenta (Braun), Dendritobilharzia loossi Skrjabin);

En Prusse Orientale, par Szidat (1928) (Bilharziella polonica Kow.) ;

En Suède, par Odhner (1910-1912) (Gigantobilharzia acotylea Odhner et Ornithobilharzia intermedia Odhner) ;

En Angleterre, par Price (1929) (Gigantobilharzia acotylea Odhner) ;

En France, dans les Dombes, par Marotel (1908) (Ornithobilharzia turkestanicum Skrjabin);

En Italie, par Parona et Ariola (1896), Parona (1899) (Ornithobilharzia kowalewskii Parona et Ariola);

En Sardaigne, par Sanfelice et Loi (1896), Bertolini (1908), Alceo (1926) (Schistosoma bovis Sonsino);

En Sicile, par Grassi et Rovelli (1888), Barbagallo (1899) (Schistosoma bovis Sonsino).

Les larves de ces trématodes sont des cercaires à queue bifur- quée, dont l'organisation rappelle celle des cercaires de Schistosoma ; elles ont de même été signalées assez rarement en Europe. Parmi elles nous pouvons citer :

La cercaire de Schistosoma bovis Sonsino, découverte récemment par E. Brumpt en Corse, dans Bullinus contortus (1929-1930); la cercaire de Bilharziella polonica Kow., récoltée par Szidat, en Prusse Orientale, dans Planorbis corneus (1929); enfin, Cercaria ocellata La Valette. Celle-ci fut trouvée, pour la première fois, par La-Valette-St-Georges (1854), en Allemagne, dans Limnaea stagnalis. Elle a été signalée par Ssinitzin (1910) qui l'avait rencontrée une seule fois, en 1902, aux environs de Varsovie (Pologne), dans

AnNales de Parasitologie, $\mathrm{T}$. ViII, $\mathrm{N}^{\circ} 2 .-1^{\text {er }} \operatorname{mars} 1930$, p. 151-160. 
Limnaea stagnalis. Enfin, Dubois (1929) l'a récoltée, une seule fois aussi, près de Neuchâtel (Suisse), toujoùrs dans L. stagnalis. La cercaire décrite par Ercolani (1881) sous le nom de C. ocellata La Val. et qui provenait de Planorbis corneus, n'est vraisemblablement pas la véritable $C$. ocellata La Val.. D'après le dessin donné par Ercolani, cette cercaire serait sans doute celle de Bilharziella polo. nica Kow., décrite récemment par Szidat. Enfin, Lühe (1909) signale, sous la dénomination de Cercaria sp., une cercaire semblable à Cercaria ocellata La Val. qui aurait été trouvée une seule fois en Prusse Orientale, près de Kœnigsberg, dans Bithynia tentaculata. Cette dernière cercaire est certainement à rapprocher aussi de la larve de Bilharziella polonica.

Au cours de mes recherches sur les Trématodes, dans ces huit dernières années, j'ai rencontré seulement, à quatre reprises différentes, Cercaria ocellata La Val. dans des mollusques récoltés en France.

En juin 1922, j'ai ramassé, dans la mare de la Patte-d’Oie, à Meudon (Seine-et-Oise), une Limnaea stagnalis qui renfermait C. ocellata La Val., mais, depuis cette date, je n'ai plus jamais retrouvé cette cercaire dans cette station. Au début de septembre 1927, j'ai trouvé, dans le réservoir du canal de Bourgogne, situé près du village de Chazilly (Côte-d'Or), des Limnaea limosa qui contenaient $C$. ocellata La Val.; sur 44 limnées récoltées, il y en avait 3 qui hébergeaient ce parasite, ce qui nous donne une proportion d'infestation de $6,810 / 0$, proportion relativement forte. Je note, en passant, que, bien que dans ce réservoir il y eût ensemble des $L$. limosa et des $L$. stagnalis, seules les premières me donnèrent $C$. ocellata La Val. ; presque tous les individus de $L$. stagnalis étaient indemnes de larves de trématodes et ceux qui en renfermaient ne me montrèrent que des Tetracotyle et des kystes d'Echinostomes. En août 1928, j'ai, à nouveau, observé $C$. ocellata La Val. dans les L. limosa du réservoir de Chazilly (Côte-d'Or). A cette époque, sur $93 \mathrm{~L}$. limosa récoltées, j'en ai trouvé 8 qui renfermaient cette cercaire. La proportion des limnées infestées par ce parasite était donc en 1928 de $8,600 / 0$, c'est-à-dire un peu plus forte que l'année précédente. Pas plus qu'en 1927, je n'ai rencontré dans cet endroit une $L$. stagnalis qui renfermât $C$. ocellata La Val..

En août 1929, j'ai recherché cette cercaire dans les mollusques du réservoir de Chazilly, mais sans succès, car ces animaux étaient rares. Les grands froids, survenus au cours de l'hiver 1929, avaient provoqué la mort de beaucoup de mollusques; de plus, durant l'été suivant, le réservoir avait été vidé en grande partie pour ali- 
menter le canal de Bourgogne; ses bords vaseux étaient presque impraticables, ce qui rendait la récolte des limnées difficile, pour ne pas dire impossible. En septembre 1929, j'ai rencontré cependant $C$. ocellata La Val. dans une $L$. stagnalis provenant de l'étang de Breuil, situé aux environs d'Arnay-le-Duc (Côte-d'Or).

Il est important de constater que Limnaea limosa peut servir d'hôte à Cercaria ocellata La Val., car c'est la première fois, à ma

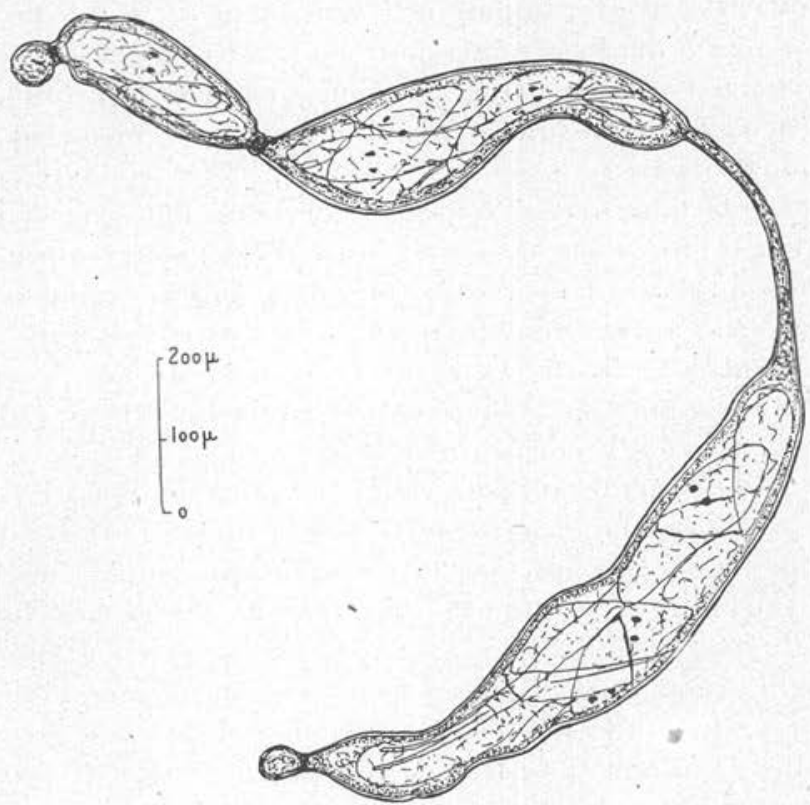

Fig. 1. - Sporocyste de Cercaria ocellata La Val.

connaissance, que ce mollusque est signalé comme hébergeant cette cercaire.

Les sporocystes (fig. 1), qui engendrent cette cercaire, sont très longs et fortement entremêlés les uns dans les autres. Il est fort difficile de les obtenir au complet. J'ai réussi toutefois à en isoler qui atteignaient de $11 \mathrm{~mm}$., 6 à $23 \mathrm{~mm}$., 2 de long, taille comparable à celle des sporocystes de Strigea tarda, et encore je ne puis affirmer qu'ils étaient entiers. Ils présentent des étranglements successifs d'aspect noirâtre qui séparent des renflements brun très clair où se trouvent les cercaires. La largeur de ces renflements varie de 120 à $200 \mu$, tandis que les parties rétrécies peuvent être filiformes et présenter seulement $10 \mu$ d'épaisseur pour une longueur de $350 \mu$, ce qui explique la fragilité de pareils sporocystes. 
Dans l'intérieur d'un sporocyste examiné, j’ai observé, une fois, la présence d'un Tetracotyle.

La cercaire (fig. 2) a une longueur totale qui varie de $0 \mathrm{~mm}$., 8 à $1 \mathrm{~mm}$., 15. Son corps, allongé, très faiblement coloré en jaunâtre, mesure de 250 à $440 \mu$ de long sur 50 à $100 \mu$ de large, au niveau de la plus grande largeur qui se trouve à la hauteur de la ventouse ventrale. La queue, plus longue que le corps, est bifurquée au-delà de sa moitié. Le tronc impair est sensiblement aussi large à sa base que le corps de la cercaire' non contractée et mesure de 350 à $450 \mu$ de long. Il se rétrécit légèrement jusqu'à l'endroit où il se divise en deux branches qui sont nettement séparées de lui par une constriction. Les deux branches caudales, dont la taille varie de 240 à $300 \mu$ de long, ont à leur base de 20 à $30 \mu$ de large et se terminent par une sorte de petit cône. Elles sont munies d'une membrane ondulante très visible, placée dans leur plan médian.

La ventouse antérieure piriforme est très volumineuse. La ventouse ventrale, située en arrière de la moitié du corps, a de 25 à $35 \mu$ de diamètre. Elle est susceptible de faire fortement saillie à la surface du corps.

La cercaire présente sur sa région dorsale, un peu en avant de la moitié du corps, une paire de taches oculaires colorées en noir ou en brun très foncé, qui ont la forme d'une coupe dans laquelle se trouve un petit cristallin. Le diamètre de ces taches varie de 7 à $10 \mu$.

Dans l'intérieur du corps se trouvent cinq paires de cellules glandulaires, dont une bonne description a été donnée par Dubois. Parmi celles-ci, quatre paires sont situées en arrière de la ventouse ventrale et remplissent toute la région inférieure du corps. Les cellules qui composent les trois paires postériéures possèdent un noyau assez gros et ont un aspect plus finement granuleux que celles des deux paires antérieures. La première paire de cellules glandulaires est située juste au-dessus de la ventouse ventrale, tandis que la deuxième paire se trouve placée directement au-dessous. Chaque cellule glandulaire se prolonge, vers l'avant, par un canal qui se joint aux canaux des autres cellules glandulaires pour former, de chaque côté, un faisceau sinueux. Les divers canaux, après avoir pénétré dans la ventouse antérieure, viennent déboucher, séparément, à l'extrémité avant du corps. Dans la région où ils se terminent, on constate la présence de quelques épines assez fortes.

Comme Dubois l'a très bien indiqué, le système excréteur (fig. 3) comprend une vésicule excrétrice de faibles dimensions qui donne, de chaque còté, un canal excréteur sinueux. Celui-ci, au 
niveau du bord arrière de la ventouse ventrale, se recourbe pour donner une branche descendante externe qui se bifurque bientôt en un canal qui remonte vers la ventouse antérieure et un autre qui se dirige vers la région postérieure du corps. Un canal excréteur médian s'étend dans le tronc impair de la queue. Il se bifurque

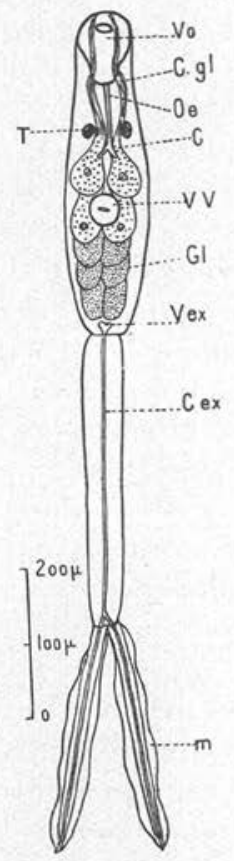

FIg. 2. - Cercaria ocellata La Val. $V O$, ventouse orale; $V V$, ventouse ventrale; C. $g l$, canaux glandulaires; Gl., cellules glandulaires; $\sigma E$, œsophage ; $C$, crecums; $V$. ex, vésicule excrétrice; $C$. ex, canal excréteur ; $m$, membrane ondulante; $T$, taches oculaires.

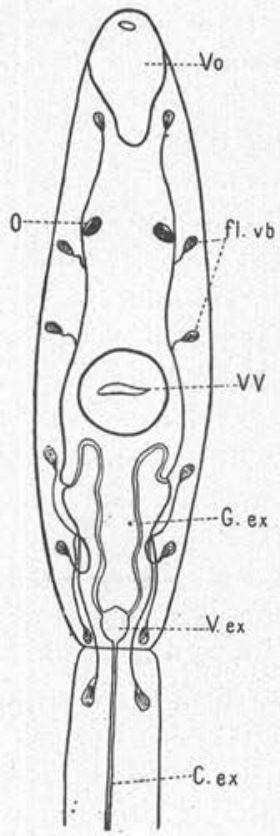

FIG. 3. - Schéma du système excréteur de Cercaria ocellaía La Val. VO, ventouse orale; $V V$, ventouse ventrale; fl. $v b$., flammes vibratiles; $C$. $e x$, canaux excréteurs; $V$. ex, vésicule excrétrice; $O$, taches oculaires.

près de la fourche caudale pour donner deux canaux situés sur la ligne médiane de chaque branche caudale à l'extrémité desquelles ils se terminent. Comme Ssinitzin et Dubois, j'ai constaté qu'il y avait sept paires de flammes vibratiles dont une seule située à la base de la queue. Le système excréteur de la cercaire de Bilharziella polonica est tout à fait semblable à celui de $C$. ocellata La Val..

Le tube digestif, très difficile à bien voir, est formé par un œesophage très étroit qui se bifurque en arrière des yeux en deux cæcums 
courts. Ce tube digestif, dépourvu de pharynx, est semblable à celui des cercaires de Schistosoma.

Cercaria ocellata La Val. se distingue de la cercaire de Bilharziella polonica par sa taille plus grande (C. ocellata: $800 \mu$ à $1 \mathrm{~mm}$., 15 de long ; cercaire de $B$. polonica : 500 à $600 \mu$ ) et par la forme de sa queue qui, à sa base, possède une largeur à peu près égale à celle du corps, alors que chez la cercaire de Bilharziella polonica, la queue, élargie en coupe à la base, enserre la partie postérieure du corps.

Les chiffres que j'ai obtenus dans les mensurations que j'ai faites sur C. ocellata La Val. sont intermédiaires entre ceux donnés par La Valette et ceux indiqués par Dubois. Il n'y a pas à signaler de différences de taille chez les $C$. ocellata provenant soit de $L$. limosa, soit de L. stagnalis.

Dès sa sortie hors de la limnée, $C$. ocellata La Val. nage dans l'eau ambiante, mais ne vient pas se cramponner à la surface libre du liquide, comme le fait la cercaire de Bilharziella polonica, d'après Szidat. Après quelques instants de nage libre, C. ocellata se fixe sur les parois du récipient à l'aide de sa ventouse ventrale qui est fortement pédiculée. La surface dorsale de la cercaire est alors concave et la queue est redressée sensiblement dans le prolongement de la partie inférieure du corps, les deux branches de la fourche étant rapprochées l'une de l'autre. La queue peut du reste être rabattue plus complètement sur le corps, comme Ssinitzin l'a indiqué. La cercaire est alors immobile en apparence, mais son corps est parcouru par des contractions. Si l'eau qui renferme des C. ocellata ainsi fixées vient à ètre agitée, immédiatement on voit les cercaires reprendre leur nage, puis, au bout de quelque temps, elles viennent à nouveau se fixer aux parois du récipient. D'après Ssinitzin, ces cercaires resteraient ainsi immobiles durant 24 heures, temps au bout duquel elles mourraient. J'ai pu constater, à plusieurs reprises, que la durée de la vie de $C$. ocellata est bien supérieure à celle indiquée par Ssinitzin. J'ai, en effet, conservé, durant 60 à 65 heures, de ces cercaires bien vivantes et nageant encore librement; une fois même, j'ai pu observer quelques C. ocellata nageant encore 69 heures après être sorties d'une Limnaea limosa.

Les résultats que j'ai obtenus se trouvent vérifiés par ceux de Dubois qui a constaté que $C$. ocellata tombe sur le fond des vases après 62 heures de vie libre. Une fois tombée sur le fond des récipients, $C$. ocellata se déplace encore faiblement en rampant, après avoir le plus souvent détaché sa queue, puis elle finit par mourir. Cercaria ocellata présente donc une résistance très grande qui la différencie nettement de la cercaire de Bilharziella polonica qui, 
d'après Szidat, ne vit pas au delà de 24 heures. Dans les cultures qui m'ont servi pour établir la durée de la vie de $C$. ocellata, je n'ai jamais renouvelé l'eau au cours des 60 à 69 heures pendant lesquelles les cercaires ont vécụ. Je considère que, dans la nature, la résistance de $C$. ocellata doit être supérieure à celle que j'ai constatée, car les conditions de vie sont beaucoup plus favorables. L'aération de l'eau est assurée par la présence des plantes aquatiques et les fermentations sont le plus souvent absentes.

J'ai cherché à obtenir expérimentalement le cycle évolutif de cette larve de trématode. Ssinitzin, en 1902, avait mis cette cercaire au contact d'invertébrés et de poissons mais n'obtint aucun résultat. J'ai constaté qu'en effet il n'y avait absolument aucune attraction de $C$. ocellata par des mollusques, des poissons, des larves de tritons, des têtards de grenouilles, etc., mis dans de l'eau qui renfermait cette cercaire.

D'après son anatomie, qui rappelle beaucoup celle des larves de Schistosoma, C. ocellata doit appartenir au cycle évolutif d'un trématode parasite dans les vaisseaux sanguins d'un vertébré supérieur et très probablement d'un oiseau. J'ai alors fait des expériences à l'aide de jeunes poulets et de canards adultes, seuls oiseaux que j'ai pu me procurer à cette époque à la campagne.

L'eau renfermant $C$. ocellata était mise au contact de la peau du ventre ou de la partie inférieure de l'aile des oiseaux après que les plumes et le duvet avaient été enlevés. C'est surtout sur la peau de l'aile que j'ai fait mes expériences car les poulets que je possédais, étant jeunes, avaient les ailes presque complètement dépourvues de duvet et de plumes sur leur face inférieure. D'autre part, je pouvais suivre plus commodément l'action des cercaires sur la peau des ailes.

Sur la surface inférieure déplumẹe de l'aile d'un poulet ou d'un canard, j'ai donc placé de l'eau qui renfermait de nombreuses C. ocellata nageant activement. J'ai alors constaté que, très peu de temps après avoir été mises au contact de la peau de l'oiseau, les Cercaria ocellata se fixent et détachent leur queue. J'ai ainsi souvent pu observer que, de une à deux minutes au plus après la mise au contact de la peau de l'oiseau avec l'eau qui contenait $C$. ocellata, je trouvais de nombreuses queues libres qui continuaient à se déplacer dans l'eau (j'en ai compté à plusieurs reprises de 150 à 200 après diverses expériences). Ces queues ne tardent du reste pas à mourir et à se décomposer. Elles cessent tout mouvement de un quart d'heure à une demi-heure après leur séparation d'avec le corps de la cercaire et quatre heures après elles sont déjà fortement désorganisées. La fixation des cercaires sur la peau de l'aile est plus 
rapide que sur celle du ventre. Je dois noter que presque toutes les. cercaires se fixent dans le temps minimum.

D'après mes expériences, il n'y a aucun doute qu'il existe une attraction manifeste de certains oiseaux (canard, poulet) pour Cercaria ocellata et que cette dernière pénètre directement à travers la peau dans l'hòte définitif. Dès que j'observais des queues s'agitant. librement dans l'eau mise au contact de l'oiseau, je cherchais à obtenir des cercaires dépourvues de queue, en raclant la surface de la peau, mais je n'en ai jamais récolté. De même, l'aile examinée au microscope ne montrait à sa surface aucune trace de cercaires. La pénétration de $C$. ocellata à travers la peau de l'aile doit donc se. faire très rapidement en une ou deux minutes, peut-être moins. Ceci n'a rien qui puisse surprendre car on connait des cas de pénétration très rapide de cercaires dans un hôte. Dubois indique que la pénétration de Cercaria letifera Fuhrmann dans le corps de poissons mis à son contact est très prompte. Il s'exprime ainsi : "La fixation est instantanée : la cercaire s'implante comme une flèche dans la peau et pénètre très rapidement. Au bout d'une vingtaine de secondes, le corps est enfoui et la queue se détache aussitòt. "Je crois que les cercaires de Schistosoma doivent également pénétrer dans leurs hôtes, à travers la peau, avec une très grande rapidité.

Après l'application de l'eau renfermant $C$. ocellata, on constate que la peau de l'oiseau présente de nombreux points rouges, principalement aux endroits d'insertion des plumes. Si l'on fait plusieurs applications d'eau avec des cercaires, sur la même surface de peau, à des intervalles assez rapprochés, on observe que l'épiderme jaunit puis se desquame abondamment. $\mathrm{Si}$ on répète plusieurs fois l'expérience dans une même journée, il se forme, sur la surface où les cercaires ont pénétré, une croûte épaisse de couleur jaune.

Certains des oiseaux en expérience sont morts quelques jours après avoir été infestés, d'autres furent conservés durant trois semaines, puis sacrifiés. A l'autopsie de ces différents sujets, je n'ai malheureusement rien retrouvé dans les vaisseaux sanguins. Ces expériences ayant été faites à la campagne, où je n'avais qu'une installation de fortune très défectueuse, il est possible que l'infestation ait réussi mais que je n'aie pas pu la constater, faute de moyens matériels. Même si le canard et le poulet ne sont pas des hôtes favorables pour le développement complet de $C$. ocellata, mes expériences montrent, d'une façon claire, que cette cercaire est susceptible de se fixer sur la peau des oiseaux, puis de pénétrer à travers elle dans l'intérieur du corps. C'est surtout aux points d'inser- 
tion des plumes que l'on constate des zones d'irritation après l'application de l'eau contenant les cercaires. Cercaria ocellata s'infiltre dans la peau du canard et du poulet d'une façon tout à fait semblable à celle indiquée par Szidat pour la cercaire de Bilharziella polonica. En effet, celle-ci pénètre dans la peau des ailes, aux endroits dénudés, et rampe le long des axes des plumes. Il est intéressant de constater que ces deux cercaires qui possèdent une organisation très voisine se comportent de la même façon pour opérer leur pénétration dans leur hòte définitif.

\section{RÉsumé}

Cercaria ocellata La Val. a été rencontrée par l'auteur, en France, dans la mare de la Patte d'Oie à Meudon (Seine-et-Oise), dans le réservoir de Chazilly (Côte d'Or) et dans l'étang de Breuil (Côte d'Or). Les Mollusques dans lesquels cette cercaire a été trouvée sont Limnaea stagnalis et Limnaea limosa. C'est la première fois que $L$. limosa est indiquée comme hôte de $C$. ocellata La Val..

Cette cercaire peut vivre à l'état libre durant une soixantaine d'heures. L'auteur a pu montrer expérimentalement que $C$. ocellata La Val. pénètre très rapidement à travers la peau du poulet et du canard, de la même manière que la cercaire de Bilharziella polonica Kow., mais les stades ultérieurs à la pénétration n'ont pas été retrouvés lors de l'autopsie des sujets mis en expérience.

\section{Bibliographie}

Alceo (G.). - La Bilharzia crassa negli ovini sardi. Clinica veterinaria, XLIX, 1926, p. 78-81.

Barbagallo (P.). - Contributo alla studio della Bilharzia crassa in Sicilia. Archives de Parasitologie, Il, 1899, p. 277-285.

Bertolisi (G.). - Osservazioni sulla Bilharzia crassa (Schistosomum bovis) e sulle alterazioni da essa prodotte. Clinica veterinaria, XXXI, 1908, p. 1-67.

Brumpt (E.). - Cycle évolutif du Schistosoma bovis (= Bilharzia crassa). Infection spontanée du Bullinus contorlus en Corse. C. R. Ac. Sc., CLXXXiX. 1929 , p. $879-881$. (Note préliminaire).

- Cycle évolutif complet de Schistosoma bovis. Infection naturelle en Corse et infection expérimentale de Bullinus contortus. Annales de Parasitologie, VIII, 1930, p. 17-50.

Dubors (G.). - Les Cercaires de la région de Neuchâtel. Thèse de doctorat de l'Université de Neuchâtel, 1929, 177 p.

Ercolani (G.). - Deli' adattamento della specie all' ambiente. Nuove ricerche sulla storia genetica dei Trematodi. Mémoires Ac. Bologne, II, 1881, p. 239-334. 
Grassi (B.) et Rovelur (G.). - La Bilharzia in Sicilia. Atti della R. Ac. dei Lincei (4), IV, 1888, p. 799.

Kowalewski (M.). - Studya helmintologiczne, III. Krakow, 1895, p. 1-30. (Extrait du tome XXXI, Rozpraw Wydzialu mat. Ak. Umiej. Krakowie).

- Studya helmintologiczne, IV. Bull. Inter. Ac. Sc. Cracovie, 1896, p. 145-148.

La Valette St-Georges (A. von). - Symbolae ad Tremalodum evolutionis Historiam. Berolini, 1884 , p. 1-40.

Lüне (M.). - Die Süsswasserfauna Deutschlands, Heft 17, Trematoden, 1909, p. 1-217.

Marotel (G.). - Existence de la bilharziose bovine en France. Recueil de Méd. vétér., LXXXV, 1908, p. 119-122.

OdhNer (T.). Gigantobilharzia acotylea, n. g. n. sp. ein mit den Bilharzien verwandter Blutparasit von enormer Länge. Zool. Anz., XXXV, 1910, p. $380-385$.

- Zum natürlichem system der digenen Trematoden, V. Zool. Anz., XLI, 191̣2, p. 54-71.

Parona (C.) et Ariola (V.). - Bilharzia kowalewskii n. sp. nel Larus melanocephalus. (Nota preventiva). Atti Soc. Ligustica Sc. nat. e Geo., Genova, VII, 1896, p. 114-116.

Parona (C.). - Catalogo di Elminti raccolti in Vertebrati dell Isola d'Elba dal doti. Giacomo Damiani. Boll. dei Musei Zool. e Anat. comp. R. Univ. Genova., n॰77, 1899, p. 1-16.

Price (E.-W.). - A synopsis of the Trematode family Schistosomida with description of new genera and species. Proc. U. S. National Mus., LXXV, 1929, p. 1-39.

SAnfelice (F.) et Loi (L.). - Ueber das Vorkommen von Bilharzia crassa Sonsino in der Leber von Rindern in Sardinia. Centralbl. f. Bakt., I Abt., XX, 1896, p. 305 .

Ssinitzın (D.-Th.). - Studien über die Phylogenie der Trematoden. 2. Bucephalus v. Baer und Cercaria ocellata De la Val. Zeitschr. wiss. Zool., LXXXXIV, 1910, p. 299-325.

Szidat (L.). - Studien an einigen seltenen Parasiten der Kurische Nehrung. Zeitsch. f. Parasitenkunde, I, 1928, p. 331.

- Zur Entwicklungsgeschichte des Blut-Trematoden der Enten, Bilharziella polonica Kow. I. Morphologie und Biologie der Cercaria von Bilharziella polonica Kow. Centralbl. f. Bakt. Orig., CXI, 1929, p. 461-470. 